\title{
ASSESSMENT OF POLYMERIZATION SHRINKAGE OF DIFFERENT BULK-FILL RESIN COMPOSITES
}

\author{
Ibrahim El Dossoky Basha ${ }^{1 *}$, Hamed Ibrahim Mohamed ${ }^{1}$, Mohamed Ahmed Wakwak ${ }^{1}$
}

\begin{abstract}
Objectives: This research was designed to evaluate the effect of polymerization shrinkage of different bulk-fill resin composites. Materials \& Methods: Ninety extracted molars were prepared as specimens by having a box shape proximal cavity for restoration of composite resins. The specimens were divided for three main groups $(n=30)$, according to the resin composite materials used (sonic fill, flowable and packable bulk fill). Profile projector was used for assessment of polymerization shrinkage. Results: There is no significant difference of variables. Sonic fill composite resin has lower polymerization shrinkage than flowable bulk fill composite and packable bulk fill composite. Conclusions: success of composite depends mainly on the material polymerization shrinkage as when the shrinkage decrease mostly the postoperative problems decrease.
\end{abstract}

KEYWORDS: Sonic fill composite resin, Polymerization shrinkage, Marginal adaptation, Bulk fill composite

\section{INTRODUCTION}

The use of composite resins is widely used as a direct esthetic restoration material. The polymeric matrix contains a variety of high molecular weight, mainly based on bis-GMA,ATEGDMA and glycidyl methacrylate. Filler particles account for up to 86 per cent of the material's weight and 71 per cent of its volume, and the types used in composites include quartz, borosilicate glass, and aluminosilicate glass. The monomer matrix is extremely viscous. Therefore, diluent monomers are added to improve functionality and flowability. bis-GMAdemonstrates a higher mass than dysfunctional monomers such as DEGMA or TEGDMA or, monomer systems, such as aromatic urethane dimethacrylates, are used in some composite resins instead of bis-GMA ${ }^{(1-4)}$.

Inadequate adaptation at the tooth/restoration interface, micro-cracking, post-operative sensitivity, micro-leakage, and secondary caries have all been linked to polymerization shrinkage stress. Changes in material formulations and filling techniques, aimed to minimize volumetric contraction and shrinkage stress, have been the mainstreams for reducing the development of residual stresses ${ }^{(5)}$.

To decrease the effects of polymerization, shrinkage enhances marginal adaptation, reduces marginal leakage, decreases cuspal, makes the cusps more resistant to subsequent fracture, and decreases postoperative sensitivity, incremental placing technique and bulk-fill technique were developed. However, the theory that an incremental placement reduces the stress effects of resin-based composite (RBCs) is still debated. It is stated that incremental curing neither eliminates the gingival contraction gap (micro-leakage) nor improves the marginal adaptation of the restorations. The bulk-fill technique could

1. Lecturer, Operative Department, Faculty of Dental Medicine, Al-Azhar University, Boys, Cairo

-Corresponding author: IbrahimBassha.209@azhar.edu.eg 
allow the placement of larger increments of composites in a single application. Over a relatively short duration, many bulk-fill composites resin has been used in increment depths between 4-5 mm. Bulk-fill RBC restorative materials can be categorized into high-viscosity or low-viscosity, light or dual-cured ${ }^{(6-8)}$

Concerning the polymerization initiation system, major modifications have been done, giving a new polymerization initiation booster called Ivocerin, which is described as an initiator system based on Germanium of greater reactivity of absorption of 400 to $450 \mathrm{~nm}$. As a result, the amount of filling of these resins has been observed to be lower than that of conventional microhybrid and nanohybrid RBCs, with filling percentages comparable to flowable RBCs in volume but higher in weight ${ }^{(9-10)}$.

Sonic Fill is a high-viscosity bulk-fill resin RBC that uses sonic vibration to become low-viscosity. Because of the flowable properties caused by sonic vibrations, the material may become very close to the cavity walls. That material is made up of a densely packed composite resin and modifiers activated by sonic energy generated by a specially designed handpiece ${ }^{(11-12)}$. This study evaluates the adaptation of sonic fill resin composite with flowable and packable bulk-fill composite resin.

\section{MATERIAL AND METHODS}

A total number of 90 freshly extracted, intact human molars were selected for this study. Class II box type cavities were prepared on one proximal surface of each tooth using straight fissure carbide bur number 57 size 010(Brassler, USA) rotating at high speed with air/water cooled handpiece (PANA MAX, NSK, Japan) ${ }^{(13)}$. The dimensions of the cavity were standardized at $4 \times 2 \times 5 \mathrm{~mm}$ (buccolingual, mesiodistal \&occluso-gingival depth respectively). A new bur was used every five cavity preparations and dimensions of each cavity preparation were checked using graduated periodontal probe.

\section{Application of Adhesive Systems:}

The etchant gel applied for 15 seconds (The etchant used is Scotch bond 3M ESPE). Then vigorously air-dried for removal of excess moisture. Adper Single Bond 2 Adhesive (3M ESPE) was applied onto the cavity wall surfaces with a disposable brush for 20 seconds in a scrubbing motion, gentle airstream was used to spread the bond and then light-cured for 10 seconds using LED light curing unit (LED Ivoclar Vivadent, Schaan, Liechtenstein, Germany) with light intensity (500-800 Mw/ $\mathrm{cm} 2$ ) according to the manufacturer instruction. Standardized tofflemire matrix holder (Town brothers Pvt., Pakistan) with No.1 metal matrix band was applied before resin composite packing to prevent overhanging of the restorative material.

\section{Application of resin composite materials:}

1. Application of sonic fill composite: starting from the bottom of the cavity until complete filling of the cavity using SonicFill handpiece (Kerr Corporation, Orange CA 92867, U.S.A). the handpiece was used under air pressure between 30-50 psi ( 2-3.4 Bar), The middle speed for the application was used (where No.1 is the slowest, No.5 is the fastest).

2. Application of flowable bulk-fill (SureFil @ SDR Bulk-fill flowable composite, Dentsply):was placed in the deepest part of the preparation, with the tip kept close to the surface. To avoid the entrapment of air, the tip of the extruder is raised above the dispensed material as the material is extruded until the cavity is entirely filled.

3. Application of packable bulk-fill (Filtek Bulkfill packable composite $3 M$ ESPE): filled in the cavity as a one-part and condensed with the composite applicator and well packed by ball burnisher from the occlusal surface.

II. Light curing of the composite Restorations: All restorations were cured of all surfaces for 20 seconds according to the manufacturer instruction with zero distance from each surface. 
III. Finishing procedure: As a final step, the restorations finished using 15 um grit composhape finishing diamond stone (Intensiv, Viganello-Lugano, Switzerland) then polished using a Sof-Lex Finishing disc (3M ESPE,St. Paul,MN,USA).

The teeth were stored in tightly sealed labelled containers with distilled water at room temperature and it was changed every day. That was done for the storage period of either 24 hours, 3 months and 6 months.

The profile projector (Mitutoyo PJ-A3000, Japan. Designed in 2012) was used for measuring the linear polymerization shrinkage. The measure was taken before and after the application of the restorative materials. Before measuring, indicators (plastic indicators used to indicate the outline of the cavity) were added to buccolingualy from the proximal surface such that it can be seen inside the profile projector. The next step is measuring the dimensions before application of the restorative materials then measuring again after application of the restorative materials.

For parametric data, a one-way ANOVA and Tukey's posthoc tests were used to compare polymerization shrinkage for three composite types. The corresponding statistical significance was measured.

\section{RESULTS}

\section{Polymerization shrinkage}

Packable Bulk-fill resin composite demonstrated the highest mean polymerization shrinkage $(p<0.05)$. There was no statistically significant difference between Sonic Fill and Flowable Bulkfill composites; both showed the lowest mean polymerization shrinkage values, as displayed in Table 1.

TABLE (1) Descriptive statistics and results of the one-way ANOVA test for three composite types

\begin{tabular}{ccccccccc}
\hline \multirow{2}{*}{ Composite type } & Mean & SD & Median & Minimum & Maximum & $\begin{array}{c}\text { Lower } \\
\text { bound }\end{array}$ & $\begin{array}{c}\text { Upper } \\
\text { bound }\end{array}$ & $P$-value \\
\hline Sonic Fill & 6.7 & 0.5 & 7.0 & 6.0 & 7.0 & 6.4 & 7.0 & $<0.05^{*}$ \\
Flowable Bulk-fill & 8.5 & 0.5 & 8.5 & 8.0 & 9.0 & 8.1 & 8.9 & $>0.05$ \\
Packable Bulk-fill & 34.7 & 2.9 & 34.5 & 31.0 & 39.0 & 32.6 & 36.8 & $<0.001^{* *}$ \\
\hline
\end{tabular}

There was a statistically significant difference between Sonic Fill and Flowable Bulk-fill composites; both showed the lowest mean polymerization shrinkage values, while, packable composite show lower values in (figure 1)

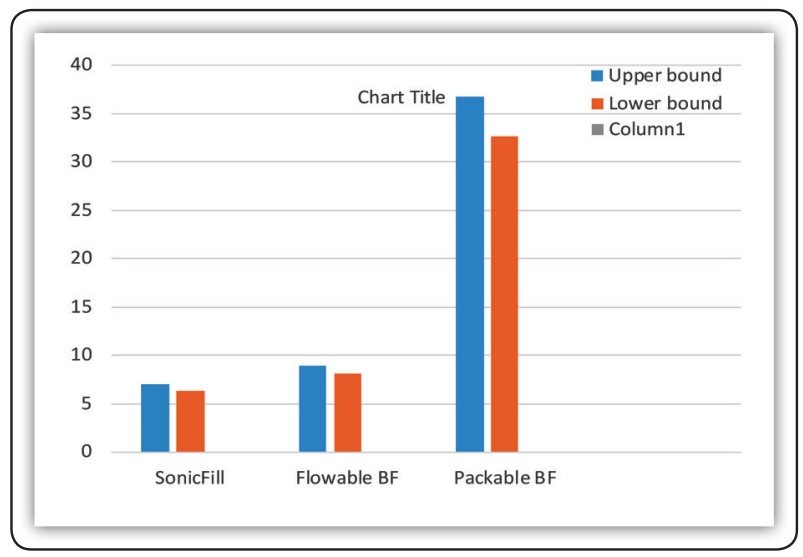

FIG (1) Bar chart representing Descriptive statistics and results of the one-way ANOVA test for three composite types 


\section{DISCUSSION}

Polymerization shrinkage is the commonest cause of failure of direct posterior composite restorations, occurring because a polymer network results from the conversion of monomer molecules. This reaction allows for the exchange of Van der Walls spaces into covalent bond spaces, creating contraction stresses and microleakage ${ }^{(14-18)}$. The problems of resin composite shrinkage usually solved using the incremental placement technique. This is a sensitive technique, so most clinicians used the bulkfill technique to decrease time. Most bulk-fill resin strives a minimum $4 \mathrm{~mm}$ depth of cure. This was accomplished by making the resin more responsive to light activation. Utilizing stress-relieving technology and new photo-initiators diminish internal stress by lowering polymerization shrinkage ${ }^{(19-22)}$.

The profile projector was used for measuring the linear polymerization shrinkage, which show no significant difference between sonic fill and packable bulk-fill while flowable bulk-fill resin composite has a greater polymerization shrinkage. The packable bulk-fill showed a polymerization shrinkage value lower than SDR flowable bulk-fill, which may be attributed to its Bis-GMA and BisEMA containing molecule. Two aliphatic carbon with double bonds and six aromatic double carbon bonds minimize contraction due to the smaller molecular weight of these monomers, while the TEGDMA and the UDMA of flowable ones have two aliphatic carbon double bonds ${ }^{(23-27)}$.

Packable bulk-fill contains two novel methacrylate monomers that, in combination, act to lower polymerization stress. One monomer, a high-molecular-weight aromatic urethane dimethacrylates (AUDMA), decreases the number of reactive groups in the resin. This helps to moderate the volumetric shrinkage as well as the stiffness of the developing and final polymer matrix, both of which contribute to the development of polymerization stress. The second unique methacrylate represents a class of compounds called addition-fragmentation monomers (AFM). During polymerization, similar to other methacrylate, AFM reacts with the developing polymer, forming cross-links between adjacent polymer chains, relieving stresses while the polymer's physical properties are preserved. The less polymerization shrinkage on sonicfill composite resin attributed to its consistency that is like a flowable when it is being placed, and then it has the properties of a hybrid after it polymerizes ${ }^{(18)}$. Sonicfill contains a proprietary rheological modifier that reacts to sonic energy from the handpiece and causes the viscosity to drop $87 \%$ during extrusion. This viscosity drop allows the SonicFill composite to rapidly flow into the cavity, allowing intimate adaptation of the composite to the cavity walls. It also displays a more gradual viscosity buildup than conventional resin composites when shear stress is removed ${ }^{(22-25)}$.

Another explanation; As the filler content is $83.5 \%$ by weight and $69 \%$ by volume, so greater incorporation of filler particles result in an increase in the modulus of elasticity of a material which reduces the amount of organic matrix in the composite and favors a reduction in the polymerization shrinkage but further evidence is needed using non-invasive assessment technique ${ }^{(28)}$.

The polymerization shrinkage results of the current study were in agreement with Hahnel et al.(25) who found that sonic fill resin composite has less shrinkage than other bulk-fill composites due to its characteristics, such as the organic matrix's composition, the type of photo-initiator, and the size and filler volume, have more satisfactory mechanical properties.

On the other hand, there was disagreement with this result with Rodriguez et al. ${ }^{(29)}$ who found that sonic fill composite resin has great volumetric changes as it contains a diluted trimethylene glycol dimethacrylate monomer and a rheological modifier to support the enhancement of the flowability of the resin paste with ultrasound, although these components of the resin matrix may contribute to increased volumetric shrinkage. 
With the limitation of in vitro studies, extended storage time is required to obtain more information. Also, clinical studies are needed in the future to assess the effect of the oral environment on the efficacy of it.

\section{CONCLUSIONS}

According to the circumstances of this study, polymerization shrinkage of flowable bulk-fill resin composite is still more than backable bulkfill resin composite even with the evolution of the technology of flowable bulk-fill. Future research is needed to investigate the polymerization shrinkage using more advanced techniques such as optical coherence tomography.

\section{REFERENCES}

1. Ferracane JL Resin composite - state of the art Dental Materials.2011; 27(1) 29-38.

2. Gao BT, Lin H, Zheng G, Xu YX, \& Yang JL. Comparison between a silorane-based composite and methacrylate-based composites: Shrinkage characteristics, thermal properties, gel point and vitrification point. JDent Mat.2012; 31(1) 76-85.

3. Chesterman J, Jowett A, GallacherA. Bulk-fill resinbased composite restorative materials: a review.Br Dent J. 2017;222(5):337-344.

4. Ilie N, Bucuta S, Draenert M. Bulk-fill resin-based composites: an in vitro assessment of their mechanical performance. Oper Dent 2013; 38(6): 618-625.

5. Benetti AR, Havndrup-Pedersen C, Honoré D, Pedersen MK, Pallesen U. Bulk-fill resin composites: polymerization contraction, depth of cure, and gap formation. Oper Dent. 2015;40(2):190-200.

6. Ilie N, Stark K. Effect of different curing protocols on the mechanical properties of low-viscosity bulk-fill composites. Clin Oral Investig. 2015;19(2):271-9.

7. Van Ende A, De Munck J and Van Landuyt KL. Bulk-filling of high C-factor posterior cavities: effect on adhesion to cavity-bottom dentin. Dent Mater. 2013;29(3):269-77.

8. Ilie N, Stark K. Curing behaviour of high-viscosity bulkfill composites. J Dent. 2014;42(8):977-85

9. Ibarra ET, Lien W and Casey J, Physical properties of a new sonically placed composite resin restorative material. Gen Dent. 2015;63(3):51-6.
10. Jang JH, Park SH and Hwang IN. Polymerization shrinkage and depth of cure of bulk-fill resin composites and highly filled flowable resin. Oper Dent. 2015;40(2):172-80.

11. El-Damanhoury H and Platt J. Polymerization shrinkage stress kinetics and related properties of bulk-fill resin composites. Oper Dent. 2014;39(4):374-82.

12. Gamarra VSS, Borges GA and Júnior LHB. Marginal adaptation and microleakage of a bulk-fill composite resin photopolymerized with different techniques. Odontology. 2018;106(1):56-63.

13. Moorthy A, Dowling AH and GruffertyBF. Cuspal deflection and micro-leakage in premolar teeth restored with bulk-fill flowable resin-based composite base materials.J Dent. 2012;40(6):500-5.

14. Carrilho Eunice, Abrantes Margarida and Casalta-Lopes João. Tc in the evaluation of microleakage of composite resin restorations with SonicFill.An in vitro experimental model. Open J Stom, 2012; 2, 340-347.

15. Herrero AA, Yaman P, Dennison JB. Polymerization shrinkage and depth of cure of packable composites. Quintessence International (2005) 36(1) 25-31.

16. Gundam s, Patil J, Venigalla B. Comparison of marginal adaptation of mineral trioxide aggregate, glass ionomer cement and intermediate restorative material as root-end filling materials, using scanning electron microscope: An in vitro study.JConserv Dent. 2014; 17(6): 566-570.

17. Heintze S, Forjanic M, Cavalleri A. Microleakage of Class II restorations with different tracers-comparison with SEM quantitative analysis. J Adh Dent. 2008;10:259-267.

18. Nawawy M, KoraitimL ,Abouelatta O. Marginal Adaptation of Nanofilled, Packable and Hybrid Dental Composite Resins Stored in Artificial Saliva. A J of Bio med Eng. 2012; 2(3): 105-114.

19. Leprince JG, Palin WM and Hadis MA, Progress in dimethacrylates-based dental composite technology and curing efficiency Dent Mater. 2013;29(2):139-56.

20. Tsujimoto A, Barkmeier WW, Takamizawa T, Latta MA, Miyazaki M. Depth of cure, flexural properties and volumetric shrinkage of low and high viscosity Bulk-fill giomers and composite resins. Dent Mat 2017;36:205-13

21. Rahiotis C, Kakaboura A, Loukidis M, Vougiouklakis G. Curing efficiency of various types of light-curing units. Eur J Oral Sci 2004; 112:89-94

22. Tonetto MR, BandécaMCandBarudHG .Influence of artificial aging in marginal adaptation of mixed class $\mathrm{V}$ cavities. J Contemp Dent Pract. 2013;14(2):316-9. 
23. Jacquot B, Durand JC and Farge P. Influence of temperature and relative humidity on dentin and enamel bonding: a critical review of the literature. Part 1.Laboratorystudies. JAdhes Dent. 2012;14(5):433-46.

24. Hofmann N, Siebrecht C and Hugo B. Influence of curing methods and materials on the marginal seal of class $\mathrm{V}$ composite restorations in vitro. Oper Dent. 2003;28(2):160-7.

25. Hahnel S, Henrich A and BürgersR.Investigation of mechanical properties of modern dental composites after artificial aging for one year. Oper Dent. 2010;35(4):412-9.

26. Mahmoud SH and Al-Wakeel S. Marginal adaptation of ormocer-, silorane-, and methacrylate-based composite restorative systems bonded to dentin cavities after water storage. Quintessence Int. 2011; 42(10):131-9.

27. Discacciati J, Rodrigo L and Alisson D.Discacciati J, Rodrigo L and Alisson D. polymerisation control by the addition of organophylic clays in a system BISGMA-TEGDMA. An7th Braz Cong Polymer.2015:300-02.

28. Basha E. Assessment of composite leakage using optical coherence tomography: A systematic review. Adv Clin Exp Dent. 2020;1(1):19-32. Doi: 10.21608/ACED.2020.150766

29. Rodriguez VI, Abate PF, Macchi RL. Immediate polymerisation shrinkage in light cured restorative resins. ActaOd ontológicaLatinoamericana (2006)19(1) 3-7. 